\title{
Coexisting Cerebral Salt Wasting Syndrome and Central Diabetes Insipidus in a Patient with Posterior Cerebrovascular Infarction: A Case Report
}

\author{
Mohamad A.E. Omar ${ }^{a}$ Hesham F. Kewan ${ }^{a}$ Hussein Kandeel ${ }^{a}$ \\ Ammar M.H. Shehadeh ${ }^{b}$ \\ aDepartment of Intensive Care, Hatta Hospital, Dubai Health Authority, Dubai, United Arab Emirates; \\ ${ }^{b}$ Department of Pediatric, Hatta Hospital, Dubai Health Authority, Dubai, United Arab Emirates
}

\section{Keywords}

Cerebral salt wasting syndrome - Central diabetes insipidus . Hyponatremia · Hypernatremia $\cdot$ Cerebrovascular infarction

\begin{abstract}
Background: Disorders of sodium balance are common in critically ill neurologic patients. However, the coexisting of cerebral salt wasting syndrome (CSW) and central diabetes insipidus (CDI) in such patients is rare. Early recognition of such conditions is challenging, thus making the prognosis ominous. Case Presentation: A 50-year-old male patient presented with acute posterior cerebrovascular infarction complicated by several attacks of disturbed sodium homeostasis. The first attack manifested as hypernatremia (up to $161 \mathrm{mmol} / \mathrm{L}$ ) and polyuria with high urine sodium (188 $\mathrm{mmol} / \mathrm{L}$ ) could only be explained by CSW on top of CDI. Especially the patient was not receiving any hyperosmolar or sodium-containing fluids. Serum sodium was corrected by desmopressin acetate. Later, the patient developed 2 attacks of hyponatremia (down to $119 \mathrm{mmol} / \mathrm{L}$ ) diagnosed as CSW that was treated with fludrocortisone. Finally, he developed
\end{abstract}

karger@karger.com www.karger.com/dmj

Karger $\stackrel{\text { ' }}{5}$

GOPEN ACCESS
(C) 2021 The Author(s)

Published by S. Karger AG, Basel

This is an Open Access article licensed under the Creative Commons Attribution-NonCommercial-4.0 International License (CC BY-NC) (http://www.karger.com/Services/OpenAccessLicense), applicable to the online version of the article only. Usage and distribution for commercial purposes requires written permission. hypernatremia (up to $165 \mathrm{mmol} / \mathrm{L}$ ) diagnosed as CDI and was treated with desmopressin acetate. Conclusion: Sodium hemostasis disorders require full consideration of serum electrolytes, intravascular volume state, and urine electrolytes in view of the clinical condition. Early diagnosis and administration of the proper treatment are the cornerstones of successful management.

(c) 2021 The Author(s)

Published by S. Karger AG, Basel

\section{Introduction}

Sodium dysregulation is considered one of the most urgent challenging situations in intensive care medicine. Several neurologic disorders including tumors, neurosurgeries, subarachnoid hemorrhages, and traumatic brain injuries could result in sodium abnormality [1]. The sodium dysregulation could be presented as central diabetes insipidus (CDI), cerebral salt wasting syndrome (CSW), or syndrome of inappropriate secretion of antidiuretic hormone (SIADH) [2]. CDI is usually associated with hypernatremia and polyuria, while the CSW presents with 


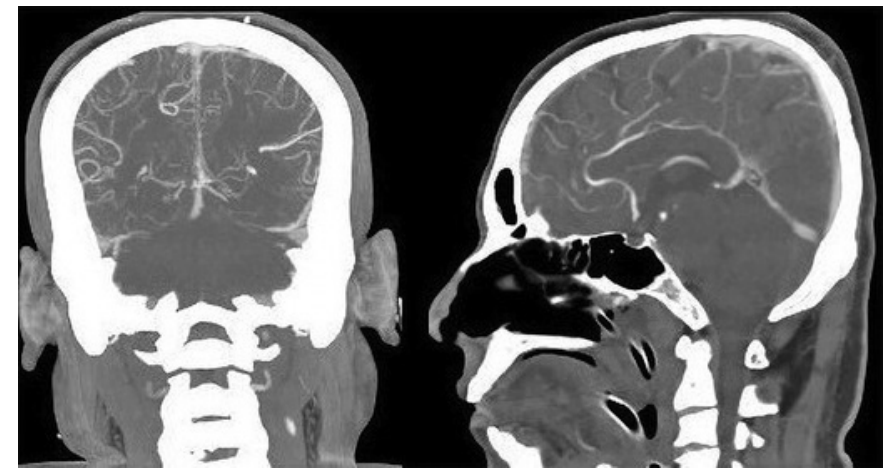

Fig. 1. A CT cerebral angiography showing occluded basilar artery just above the vertebral arteries union. CT, computed tomography.

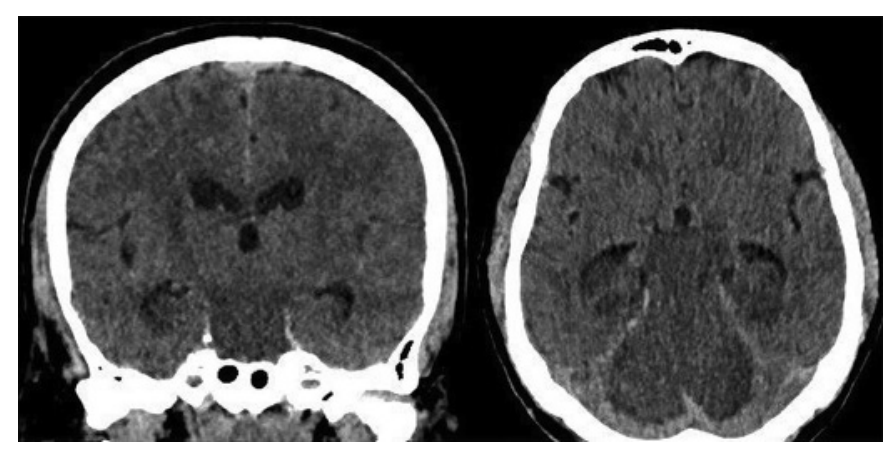

Fig. 2. A brain CT showing ischemic infarction involving the cerebellum, brain stem, occipital lobes, temporal poles, and hypothalamic nuclei with a significant cytotoxic edema. CT, computed tomography.

hyponatremia and polyuria. On the other hand, SIADH presents with hyponatremia without intravascular volume depletion $[3,4]$. The coexistence of CDI and CSW was described in a few other articles $[1,5,6]$. Yet, to our knowledge, this is the first report of coexisting CDI and CSW after cerebrovascular infarction. The management of electrolytes and fluid imbalance in such patients is usually challenging as considering the neurologic status of the patient and the poor prognosis of the original brain insult.

\section{Case Presentation}

A 50-year-old male patient presented to the hospital with a history of dizziness and vomiting twice at home after falling and head trauma. On presentation, the patient was obtunded, confused with dysarthria. Glasgow coma scale (GCS) was 13/15. Pupils were equal and reactive; the patient was not cooperative enough to perform a comprehensive neurologic examination, including the vi- sion status and visual field. However, tendon and plantar reflexes were normal. He had right parietal skin lacerations and hematoma. No other neurologic deficits were detected. Computed tomography of the brain (brain CT) showed right parietal subgaleal hematoma with no brain parenchymal abnormality. He had a medical history of old cerebrovascular ischemic stroke with no residual effect, hypertension, and polycythemia vera.

A few hours later, the patient developed further deterioration of consciousness. The GSC dropped to $9 / 15$, for which follow-up brain CT was performed.

Findings in the brain CT showed signs of acute ischemic infarctions involving cerebellar and occipital lobes bilaterally. Thrombolysis or intra-arterial thrombectomy was considered and discussed with the neurologist. However, the intervention was contraindicated due to a history of head trauma before the presentation and a history of cerebrovascular ischemia of less than 1 year.

As the GCS was plummeting, the patient was intubated, mechanically ventilated, and shifted to the intensive care unit. Further assessment by CT cerebral angiography revealed occluded basilar artery just above the vertebral arteries union denoting acute posterior circulation infarctions (shown in Fig. 1). The neurologist recommended antiplatelets, statins, and proper hydration.

The next day, neurologic examination showed unequal pupils and loss of gag reflex, so an urgent brain CT was done. Radiological progression of the ischemic infarction was prominent which included the cerebellum, brain stem, occipital lobes, temporal poles, and hypothalamic nuclei. Additionally, significant cytotoxic edema and pontine petechial hemorrhages were noticed (shown in Fig. 2). Hyperosmolar brain dehydration measures were tried; mannitol $20 \%$ was administered at a dose of $100 \mathrm{~mL}$ every $8 \mathrm{~h}$ for $48 \mathrm{~h}$. However, the patient gradually developed bilateral dilated fixed pupils and loss of most of the brain stem reflexes except he was not apneic.

During the hospital stay, the patient developed several attacks of disturbed sodium homeostasis (shown in Table 1). Initially, the patient developed polyuria, serum sodium started climbing up reaching $161 \mathrm{mmol} / \mathrm{L}$ and serum osmolality increased to $351 \mathrm{mOsm} / \mathrm{kg}$. Urine sodium level was $188 \mathrm{mmol} / \mathrm{L}$ with high urine osmolality 661 $\mathrm{mOsm} / \mathrm{kg}$. These findings were believed to be a coexisting CDI and CSW, especially since the patient did not receive high sodium fluid nor hyperosmolar therapy at least 2 days before this event.

However, CDI was the provisional diagnosis. So, desmopressin was administered to manage the significant hypernatremia and the patient showed a good response 
Table 1. The range of laboratory findings and polyuria during the episodes of CDI and CSW

\begin{tabular}{|c|c|c|c|c|c|c|}
\hline First episode & $150-161 \uparrow \uparrow$ & $321-351 \uparrow \uparrow$ & $107-188 \uparrow \uparrow$ & $584-661 \uparrow \uparrow$ & + & Combined CDI and CSW \\
\hline Second and third episodes & $116-133 \downarrow \downarrow$ & $246-283 \downarrow \downarrow$ & $87-129 \uparrow \uparrow$ & $438-564 \uparrow \uparrow$ & + & CSW \\
\hline Fourth episode & $151-165 \uparrow \uparrow$ & $322-353 \uparrow \uparrow$ & $20-44 \downarrow \downarrow$ & $215-262 \downarrow \downarrow$ & + & CDI \\
\hline
\end{tabular}

$\mathrm{Na}$, sodium; osm., osmolality; CSW, cerebral salt wasting syndrome; CDI, central diabetes insipidus.

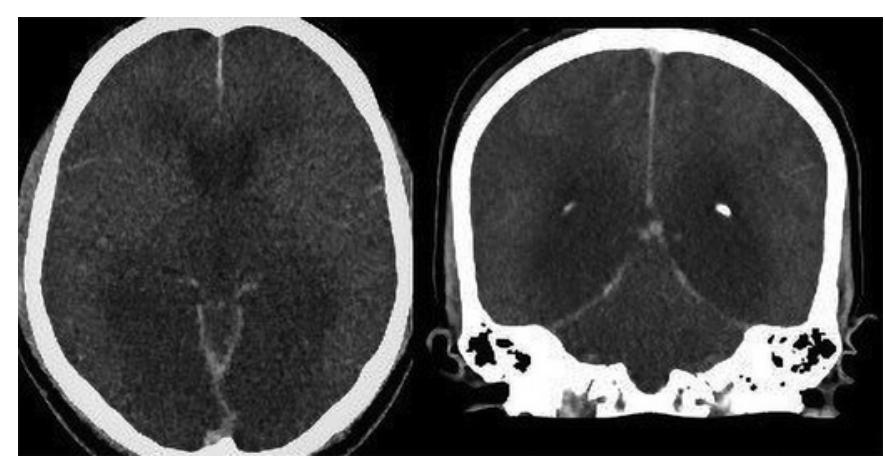

Fig. 3. A brain CT showing features of hypoxic brain injury and resolving cytotoxic edema. CT, computed tomography.

as regards urine output and correction of hypernatremia within the next few days. Follow-up brain CT revealed features of diffuse hypoxic-ischemic brain injury with resolved cytotoxic edema (shown in Fig. 3).

A few weeks later, the patient developed hyponatremia and serum hypo-osmolarity (serum sodium $119 \mathrm{mmol} / \mathrm{L}$ and serum osmolality $263 \mathrm{mOsm} / \mathrm{kg}$ ). Differential diagnosis of CSW versus SIADH was raised. However, as the patient had significant polyuria, the running diagnosis was more of CSW. Therefore, the patient was given fludrocortisone and normal saline infusion till the normalization of serum sodium. A similar episode occurred again after 2 months and was treated likewise.

Three weeks after the last CSW episode, the patient developed polyuria again. This time it was associated with hypernatremia (serum sodium $165 \mathrm{mmol} / \mathrm{L}$ ), high serum osmolality (353 mOsm $/ \mathrm{kg})$, low urine sodium (20 $\mathrm{mmol} / \mathrm{L})$, and low urine osmolality $(215 \mathrm{mOsm} / \mathrm{kg})$. These results were consistent with CDI that was managed by intravenous desmopressin acetate and optimization of intravascular volume with hypotonic solutions till serum sodium normalized.

Consequently, this patient stayed for more than 10 months, he was in a deep coma with a persistent vegeta- tive state all through the course in the intensive care unit. He developed several attacks of sepsis, renal and liver impairments. Finally, he succumbed due to septic shock and multi-organ failure.

\section{Discussion}

Abnormal sodium homeostasis in critical patients with neurologic disorders could present as CDI, CSW, or SIADH [7]. In this case report, a rare coexistence of CDI and CSW was diagnosed based on the clinical picture and laboratory investigations, including serum sodium, serum osmolality (calculated or measured), urine sodium, and urine osmolality levels. The dramatic response to the prescribed medications confirmed the diagnosis.

The CDI is relatively common. Its overall incidence is about $3.7 \%$ among neurosurgical emergencies, and $6.7 \%$ in post-craniotomy of pituitary tumor patients $[8,9]$. The CDI is usually presented with hypernatremia and polyuria (ranging from 2 up to $10 \mathrm{~L} /$ day). The degree of polyuria reflects the level of antidiuretic hormone deficiency $[3,10]$. The diagnostic criteria of CDI include hypernatremia $>145 \mathrm{mmol} / \mathrm{L}$, urine output $>200-250 \mathrm{~mL} / \mathrm{h}$, serum osmolality $>300 \mathrm{mOsm} / \mathrm{kg}$, and urine osmolality $<300 \mathrm{mOsm} / \mathrm{kg}$. Furthermore, a positive response to desmopressin acetate is a deemed confirmation for CDI diagnosis [11]. On the other hand, hyponatremia in critical patients with neurologic disorders could present as SIADH or CSW. Nevertheless, other causes may contribute to hyponatremia such as over administration of hypotonic solutions, adrenal insufficiency, and hypothyroidism. So, it is crucial to define the actual cause of hyponatremia meticulously as the subsequent treatment protocols are different [4]. CSW was first described in the 1950 s as an explanation for the occurrence of natriuresis and hyponatremia in patients with cerebral diseases [12]. It occurs in about $7 \%$ of patients presented with hyponatremia after subarachnoid hemorrhage [13]. The actual 
mechanism of CSW pathology is not clear. However, it may emerge from elevated levels of circulating natriuretic agents as well as disturbed sympathetic innervation to the kidneys which result in increased sodium excretion and contracted intravascular volume. Subsequently, low intravascular volume stimulates baroreceptors to release more antidiuretic hormone and results in hyponatremia $[14,15]$. CSW is diagnosed with hyponatremia $<135$ $\mathrm{mmol} / \mathrm{L}$, intravascular volume depletion, and negative sodium balance. Nonetheless, SIADH has a similar laboratory picture, yet no polyuria is present so, the patient is usually euvolemic to hypervolemic if compared to the hypovolemic status with CSW [16]. Differentiating between the 2 diagnoses is of paramount importance as the CSW often responds to normal saline infusion replacement and fludrocortisone therapy. In contrast, SIADH responds better to fluid restriction $[17,18]$.

In our case, the patient had massive posterior circulation cerebrovascular infarction and several attacks of abnormal sodium hemostasis. The first attack was believed to be a CDI because of polyuria and hypernatremia. Interestingly, the coexistence of CSW was raised due to the concomitant high urine sodium and osmolality with the absence of hyperosmolar therapy nor overloading with sodium-containing intravenous fluids in the preceding 2 days. This episode was managed successfully with desmopressin acetate and hypotonic fluids administration. Apparently in this episode, the CSW was masked by the coexisting CDI. The picture of CSW manifested by hyponatremia and polyuria was more evident in the subsequent 2 episodes. Fludrocortisone and optimization of intravascular volume were the cornerstones of the management of these episodes. Subsequently, the last episode was marked with hypernatremia and polyuria. CDI was the best explanation, especially with low urine sodium and improvement of the condition after desmopressin acetate administration.

\section{Conclusion}

The coexistence of CDI and CSW is a rare incidence even in patients with neurologic critical conditions. The original neurologic insult, which is cerebrovascular infarction, makes this case report unique when compared to the original etiologies of other reports. Full consideration of serum electrolytes, hemodynamic state, and urine electrolytes in view of the clinical picture is essential for proper management. Administration of the appropriate medications, for example, desmopressin acetate in CDI and fludrocortisone in CSW, in conjunction with optimization of the intravascular volume, are the cornerstones of successful management. Unfortunately, due to the rarity of this coexistence, early recognition is hard to be achieved and subsequently proper management. Additionally, the severe original neurologic condition contributes to the bleak outcome of such patients.

\section{Statement of Ethics}

Written informed consent was obtained from the patient's son for publication of this case report and any accompanying images. The consent could not be obtained from the patient as he was in a deep coma and then expired. However, as a case report, the ethical approval was not required in accordance with the Dubai Scientific Research Ethics Committee policies.

\section{Conflict of Interest Statement}

The authors have no conflicts of interest to declare.

\section{Funding Sources}

No funding related to this work.

\section{Author Contributions}

The authors were part of the treating team, Dr. M.A.E.O. and Dr. H.F.K. had written the case presentation. Dr. H.K. collected the data and extracted the images. Co-authors reviewed the case presentation and data collection. The discussion was collectively written by the full team. Dr. M.A.E.O. and Dr. A.M.H.S. prepared the final copy of the manuscript, which was reviewed and approved by the full team.

\section{Data Availability Statement}

All data generated or analyzed during this study are included in this article. Further enquiries can be directed to the corresponding author.

\footnotetext{
References $\quad 1$ Lin JJ, Lin KL, Hsia SH, Wu CT, Wang HS. Combined central diabetes insipidus and cerebral salt wasting syndrome in children. Pediatr Neurol. 2009 Feb;40(2):84-7.

2 Wu X, Zhou X, Gao L, Wu X, Fei L, Mao Y, et al. Diagnosis and management of combined central diabetes insipidus and cerebral salt wasting syndrome after traumatic brain injury. World Neurosurg. 2016 Apr; 88:483-7.
} 
3 Christ-Crain M, Bichet DG, Fenske WK, Goldman MB, Rittig S, Verbalis JG, et al. Diabetes insipidus. Nat Rev Dis Primers. 2019 Aug 8;5(1):54.

4 Rahman M, Friedman WA. Hyponatremia in neurosurgical patients: clinical guidelines development. Neurosurgery. 2009 Nov;65(5): 925-6. Discussion 935-6.

5 Costa MM, Esteves C, Castedo JL, Pereira J, Carvalho D. A challenging coexistence of central diabetes insipidus and cerebral salt wasting syndrome: a case report. J Med Case Rep. 2018 Jul 17;12(1):212.

6 Sun Lee J, Baek HJ, Kim CJ, Yang EM. Coexistence of central diabetes insipidus and prolonged cerebral salt wasting syndrome after brain tumor surgery: a case report. Child Kidney Dis. 2020 Apr;24(1):42-6.

7 Laredo S, Yuen K, Sonnenberg B, Halperin ML. Coexistence of central diabetes insipidus and salt wasting: the difficulties in diagnosis, changes in natremia, and treatment. J Am Soc Nephrol. 1996 Dec;7(12):2527-32.

8 Wong MF, Chin NM, Lew TW. Diabetes insipidus in neurosurgical patients. Ann Acad Med Singap. 1998 May;27(3):340-3.
9 Balestrieri FJ, Chernow B, Rainey TG. Postcraniotomy diabetes insipidus. Who's at risk? Crit Care Med. 1982 Feb;10(2):108-10.

10 Rose BD, Post TW. Clinical physiology of acid-base and electrolyte disorders. 5th ed. New York: McGraw-Hill; 2001. p. 758.

11 Ghirardello S, Garrè ML, Rossi A, Maghnie $M$. The diagnosis of children with central diabetes insipidus. J Pediatr Endocrinol Metab. 2007 Mar;20(3):359-75.

12 Peters JP, Welt LG, Sims EA, Orloff J, Needham J. A salt-wasting syndrome associated with cerebral disease. Trans Assoc Am Physicians. 1950;63:57-64.

13 Sherlock M, O'Sullivan E, Agha A, Behan LA, Rawluk D, Brennan P, et al. The incidence and pathophysiology of hyponatraemia after subarachnoid haemorrhage. Clin Endocrinol. 2006 Mar;64(3):250-4.
14 Spasovski G, Vanholder R, Allolio B, Annane $\mathrm{D}$, Ball S, Bichet D, et al. Clinical practice guideline on diagnosis and treatment of hyponatraemia. Nephrol Dial Transplant. 2014 Apr;29(Suppl 2):i1-39. Erratum in: Nephrol Dial Transplant. 2014 Jun;40(6):924.

15 Hannon MJ, Behan LA, O’Brien MM, Tormey W, Ball SG, Javadpour M, et al. Hyponatremia following mild/moderate subarachnoid hemorrhage is due to SIAD and glucocorticoid deficiency and not cerebral salt wasting. J Clin Endocrinol Metab. 2014 Jan;99(1):291-8. Erratum in: J Clin Endocrinol Metab. 2014 Mar: 99(3):1096.

16 Oh JY, Shin JI. Syndrome of inappropriate antidiuretic hormone secretion and cerebral/renal salt wasting syndrome: similarities and differences. Front Pediatr. 2014 Jan 22;2:146. PubMed PMID: 25657991.

17 Taplin CE, Cowell CT, Silink M, Ambler GR. Fludrocortisone therapy in cerebral salt wasting. Pediatrics. 2006 Dec;118(6): e1904-8.

18 Albanese A, Hindmarsh P, Stanhope R. Management of hyponatraemia in patients with acute cerebral insults. Arch Dis Child. 2001 Sep;85(3):246-51. 